\title{
The Kurashiki Prehospital Stroke Scale Is a Prehospital Scale That Can Predict Long-Term Outcome of Patients with Acute Cerebral Ischemia
}

\author{
Yasuyuki Iguchi Kazumi Kimura Kensaku Shibazaki \\ Yuki Sakamoto Kenichiro Sakai Shuichi Fujii Junichi Uemura \\ Department of Stroke Medicine, Kawasaki Medical School, Kurashiki City, Japan
}

\section{Key Words}

Acute stroke outcome • Emergency care - Kurashiki Prehospital Stroke Scale •

Prehospital stroke care $\cdot$ Thrombolysis

\begin{abstract}
Background and Purpose: Our aim was to confirm the clinical relationship between the Kurashiki Prehospital Stroke Scale (KPSS) scored by paramedics and favorable outcomes in patients with modified Rankin scale (mRS) scores of $0-1$ assessed 3 months after symptom onset. Methods: We enrolled patients with acute stroke and transient ischemic attack showing symptoms on admission. Paramedics transferred patients to our hospital after estimating stroke severity using the KPSS. After categorizing patients into either the mRS 0-1 group (favorable outcome) or the mRS 2-6 group (no favorable outcome), we compared the background data between the two groups. We assessed KPSS scores predictive of a favorable outcome. Multivariate regression modeling was conducted to identify factors independently associated with a favorable outcome. Results: The study cohort comprised 147 patients with a premorbid status of mRS 0-1: 69 patients (47\%) of them were in the mRS 0-1 group and 78 (53\%) in the mRS 2-6 group at the follow-up 3 months after symptom onset. The median KPSS score was lower in the mRS 0-1 group than in the mRS 2-6 group ( 1 vs. $4, p<0.001$ ). After classifying the 147 patients into KPSS tertiles with thresholds of 2 and 4, the frequency of mRS 0-1 gradually decreased with increasing KPSS score (lower KPSS, 67.2\%; middle KPSS, 47.6\%, and higher KPSS, 21.3\%; $p<0.001$ ). KPSS score $<3$ was able to predict a favorable outcome with $67 \%$ sensitivity and $71 \%$ specificity, and independently associated with $\mathrm{mRS} 0-1$ (odds ratio, 3.0; 95\% confidence interval, 1.2-7.3; $\mathrm{p}=$ 0.015). Conclusion: KPSS score $<3$ apparently presents a reasonable cutoff for predicting a favorable outcome in patients with acute cerebral ischemia.

Copyright $\odot 2011$ S. Karger AG, Basel 


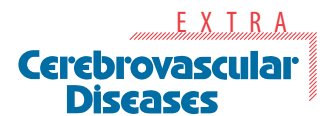

Diseases
Cerebrovasc Dis Extra 2011;1:28-35

DOI: $10.1159 / 000327154$

Published online: April 14, 2011

Iguchi et al.: KPSS and Outcome (c) 2011 S. Karger AG, Basel

www.karger.com/cee

\section{Introduction}

Since the approval of intravenous tissue plasminogen activator (IV t-PA) for ischemic stroke treatment in the United States [1], stroke care has become highly time dependent. To broaden the eligibility of patients for IV t-PA, transportation by ambulance supported by emergency medical services (EMS) is needed to minimize the duration from symptom onset to hospital arrival and decrease the time to physical examination on admission [2]. As half of the patients with signs or symptoms of acute stroke access initial medical care using an ambulance [3], appropriate identification of acute stroke by EMS paramedics possibly increases the number of patients who may benefit from IV t-PA.

To immediately transport the patient to the closest and most appropriate facility, specifically for stroke bypass surgery, two types of prehospital stroke scales are required in the field: a scale for identifying stroke and a scale for assessing stroke severity. While novel stroke screening instruments for identifying possible stroke patients (e.g. the Los Angeles Prehospital Stroke Screen and the Cincinnati Prehospital Stroke Screen) have already been used in onset scenes [4, 5], prehospital stroke severity scales (PSSSs) have gained wide attention over the last decade. PSSSs representing basic modifications of the full 15-variable National Institutes of Health Stroke Scale (NIHSS) include the Los Angeles Motor Scale (LAMS), a shortened version of the NIHSS (sNIHSS), a simple 3-item stroke scale (3-ISS), and the Kurashiki Prehospital Stroke Scale (KPSS; table 1) [6-9]. The KPSS comprises four items: level of consciousness; disturbance of consciousness; motor weakness, and language. Scores for the KPSS range from 0 to 13 , with the maximum (13 points) representing the most severe symptoms [6]. As paramedics are able to decide on appropriate transportation in accordance with stroke severity, estimating not only the possibility but also the severity of stroke will be more practical for prehospital stroke care [10]. Indeed, when stroke neurologists assessed stroke severity using PSSSs (e.g. LAMS, sNIHSS, and 3-ISS), these PSSSs were able to predict the presence of arterial occlusion $[9,11]$ and long-term outcome $[7,8]$, as well as NIHSS. However, the question of whether these assessments using PSSSs have been realistically verified in the prehospital setting remains. Our aim was thus to clarify the relationship between the KPSS score recorded by paramedics and outcome 3 months after symptom onset.

\section{Subjects and Methods}

The study cohort comprised consecutive patients with ischemic stroke or transient ischemic attack with symptoms on admission who were transferred to our facility within $24 \mathrm{~h}$ of onset between May 2007 and April 2010. The following clinical background data of all patients were collected: (1) age and gender; (2) past history; (3) duration from onset to admission; (4) NIHSS score evaluated by stroke neurologists on admission; (5) KPSS score evaluated by paramedics prior to or during transportation; (6) presence of proximal arterial occlusion and infarct size assessed by neuroimaging using computed tomography or magnetic resonance images immediately after admission; (7) use of IV t-PA; (8) premorbid status, and (9) outcome 3 months after onset.

The four items in the KPSS (level of consciousness; disturbance of consciousness; motor weakness, and language) were chosen and modified based on the full NIHSS. In accordance with the prehospital stroke care recommendations of the Japanese Society for Emergency Medicine, immediately after paramedics arrived at the scene, the condition of the patient suspected of suffering a stroke was evaluated [12]. When ischemic stroke or transient ischemic attack was considered possible, neurological severity was assessed using the KPSS [12]. Before transferring the patients to our facility, the paramedics provided precise information 


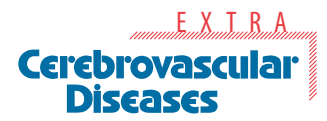

\begin{tabular}{l|l}
\hline Cerebrovasc Dis Extra 2011;1:28-35 \\
\hline $\begin{array}{l}\text { DOI: 10.1159/000327154 } \\
\text { Published online: April 14, 2011 }\end{array}$ & $\begin{array}{l}\text { @ 2011 S. Karger AG, Basel } \\
\text { www.karger.com/cee }\end{array}$ \\
\hline
\end{tabular}

Table 1. Kurashiki Prehospital Stroke Scale

Scoring (total disorder: 13 points)

Level of consciousness

Check level of consciousness

Alert

Arousal by stimulation

No response

normal: 0 points

1 point

2 points

Consciousness disturbance

Ask patient's name

Correct

Incorrect

normal: 0 points

1 point

Motor weakness (kinetic paralysis)

Ask the patient to close eyes, raise arms to the front with palms down (with gestures)

Able to keep arms raised in parallel

Able to raise arms but not to keep them in position so that they go downward

Unable to raise arms

Instruct the patient to close eyes, raise legs from the bed to the front with gestures

Able to keep legs raised in parallel

Able to raise legs but not to keep them in position so that they go downward

Unable to raise legs

right hand left hand

normal: 0 points normal: 0 points

1 point $\quad 1$ point

2 points 2 points

right leg left leg

normal: 0 points normal: 0 points

1 point 1 point

2 points 2 points

\section{Language \\ Ask the patient to say 'It's fine today' repeatedly \\ Able to clearly say it repeatedly \\ Either unclear (slurred) or abnormal \\ Silent, unable to understand linguistically}

normal: 0 points

1 point

2 points

.... points

to the staff in the emergency room. In most cases, stroke neurologists were already provided with information on the patient, including vital signs (blood pressure, heart rate, respiratory rate, and oxygen saturation), time of symptom onset, situation of the onset location, past patient history, and the KPSS score. The NIHSS score was determined by stroke neurologists on arrival of the patient at our hospital.

To achieve a precise diagnosis, we performed diffusion-weighted magnetic resonance imaging and angiography, and carotid duplex ultrasonography immediately after admission, if no contraindications were present. A large infarct was diagnosed if more than one third of the territory of the middle cerebral artery (MCA) was affected on initial diffusion-weighted imaging [13]. Proximal arterial occlusion was defined as occlusion of the main trunk of the intra- and/or extracranial artery and the corresponding symptoms. Patients diagnosed with hyper-acute stroke received IV t-PA according to previously published criteria, if eligible [13]. Outcome 3 months after onset was determined using the modified Rankin scale (mRS). A favorable outcome was defined as mRS 0 or 1 . All study protocols were approved by the Institutional Review Board of the Kawasaki Medical School (No. 587).

\section{Analysis}

After identifying cases with premorbid status mRS 0-1, we classified patients into two groups according to the postmorbid status 3 months after onset: patients in the mRS $0-1$ group had a favorable outcome and those in the mRS 2-6 group had no favorable outcome. We then categorized all patients into KPSS tertiles, in order to estimate the proportion of 
Table 2. Clinical background data of ischemic stroke patients in mRS 0-1 and mRS 2-6 groups

\begin{tabular}{lccr}
\hline & $\begin{array}{l}\text { mRS 0-1 group } \\
(\mathrm{n}=69)\end{array}$ & $\begin{array}{c}\text { mRS 2-6 group } \\
(\mathrm{n}=69)\end{array}$ & p value \\
\hline Median age (IQR), years & $70(60-76)$ & $75(66-83)$ & 0.007 \\
Males, $\mathrm{n}(\%)$ & $51(73.9)$ & $44(56.4)$ & 0.038 \\
Past history, $\mathrm{n}(\%)$ & & & \\
$\quad$ Hypertension & $43(62.3)$ & $54(69.2)$ & 0.389 \\
$\quad$ Diabetes mellitus & $17(24.6)$ & $18(23.1)$ & 0.848 \\
$\quad$ Hyperlipidemia & $17(24.6)$ & $22(28.2)$ & 0.709 \\
Smoking & $39(56.6)$ & $38(48.7)$ & 0.409 \\
$\quad$ Atrial fibrillation & $13(18.8)$ & $21(26.9)$ & 0.327 \\
$\quad$ Ischemic stroke & $7(10.1)$ & $11(14.1)$ & 0.615 \\
Time from onset to admission $\leq 3 \mathrm{~h}, \mathrm{n}(\%)$ & $43(62.3)$ & $40(51.3)$ & 0.187 \\
Median NIHSS (IQR) & $2(1-4)$ & $11(5-18)$ & $<0.001$ \\
Median KPSS (IQR) & $1(0-3)$ & $4(2-9)$ & $<0.001$ \\
KPSS <3, n (\%) & $46(66.7)$ & $23(29.5)$ & $<0.001$ \\
Infarct area of MCA >1/3 & $5 / 62(8.1)$ & $19 / 67(28.4)$ & 0.003 \\
Proximal arterial occlusion, $\mathrm{n}$ (\%) & $17(24.6)$ & $45(57.7)$ & $<0.001$ \\
Median blood glucose (IQR), mg/dl & $130(107-152)$ & $137(113-167)$ & 0.261 \\
IV t-PA, n/cases within 3 h of onset (\%) & $8 / 43(18.6)$ & $27 / 40(67.5)$ & $<0.001$ \\
\hline
\end{tabular}

$\mathrm{IQR}=$ Interquartile range.

patients with mRS $0-1$ in each tertile. The cutoff KPSS score for mRS 0-1 was then calculated by plotting sensitivity and specificity curves, and background data were compared between mRS 0-1 and mRS 2-6 groups. Significant trends were examined using the $\chi^{2}$ test and the Mann-Whitney $U$ test. Variables showing values of $p<0.1$ in univariate analyses and well-known variables related to stroke prognosis (age, atrial fibrillation, blood glucose level on admission, and baseline neuroimaging) [14-16] were considered as potential explanatory variables. Multivariate logistic regression analyses were then conducted to estimate factors independently associated with mRS $0-1$. Correlations between KPSS and NIHSS scores were analyzed using Spearman's rank correlation. Statistical analyses were performed using PASW statistics (version 18.0) software (SPSS, Chicago, Ill., USA). Values of $\mathrm{p}<0.05$ were considered significant.

\section{Results}

A total of 238 consecutive patients with ischemic stroke or symptomatic transient ischemic attack on admission (median age, 75 years; 139 men, 99 women; median NIHSS score, 6) were transferred to our hospital. Regarding the premorbid status, 147 of the 238 patients $(61.8 \%)$ were $\mathrm{mRS} 0-1$ prior to stroke onset. In these patients, an excellent correlation was evident between KPSS and NIHSS scores $(\mathrm{R}=0.728, \mathrm{p}<0.001)$.

We classified these 147 patients into two groups (table 2): the mRS $0-1$ group ( $n=69$; median age, 70 years; 51 men, 18 women) and the mRS $2-6$ group $(n=78 ;$ median age, 75 years; 44 men, 34 women). The mRS $0-1$ group was significantly younger than the mRS 2-6 group $(\mathrm{p}=0.007)$. Median NIHSS score was higher in the mRS $2-6$ group than in the mRS $0-1$ group (11 vs. 2 , respectively; $\mathrm{p}<0.001$ ). 


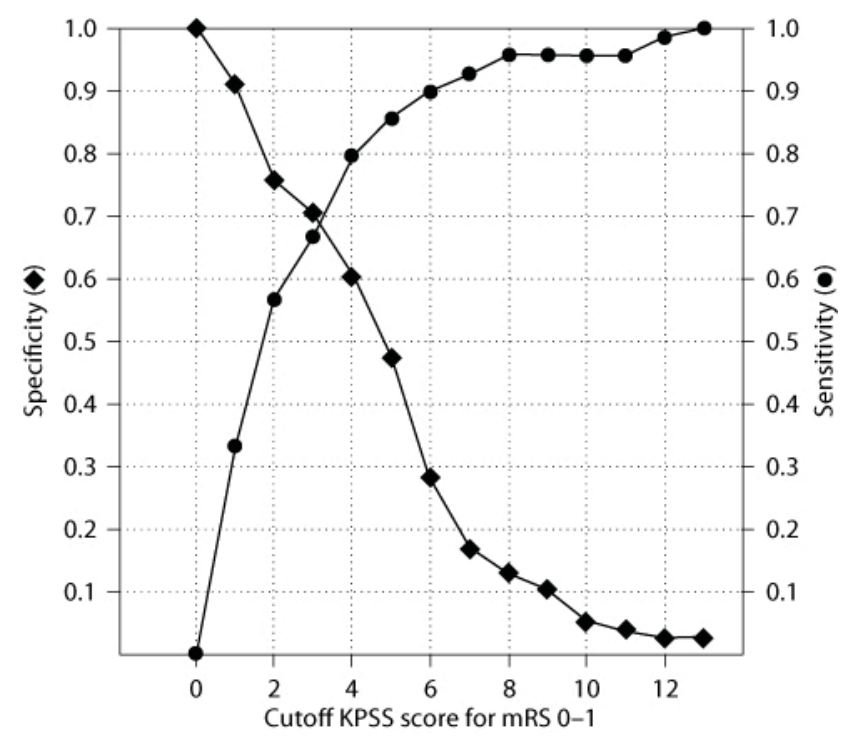

Fig. 1. Cutoff KPSS score for mRS 0-1 plotted on a sensitivity-specificity curve.

Regarding the KPSS score, after categorizing all 147 cases in KPSS tertiles using thresholds scores of 2 and 4, we compared the proportion of patients with mRS 0-1 among KPSS tertiles. Patients with mRS $0-1$ assessed 3 months after onset comprised 39 of 58 patients $(67.2 \%)$ in the lower KPSS tertile (KPSS <2), 20 of 42 patients $(47.6 \%)$ in the middle KPSS tertile, and 10 of 47 patients $(21.3 \%)$ in the higher KPSS tertile ( $<<0.001)$. Patients with lower KPSS achieved more favorable outcomes than those with higher KPSS.

Comparing background data between mRS 0-1 and 2-6 groups again, the KPSS score was significantly lower in the mRS $0-1$ group (median KPSS; 1 vs. $4, \mathrm{p}<0.001$, table 2 ). The cutoff KPSS score for predicting mRS $0-1$ at 3 months after onset was 3, with a sensitivity of $67 \%$ and a specificity of $71 \%$ (fig. 1). Patients with KPSS $<3$ were more frequently in the mRS $0-1$ group than in the mRS $2-6$ group (66.7 vs. $29.5 \%, \mathrm{p}<0.001$; table 2 ). Concerning the infarct area in patients showing ischemic lesions in the territory of the MCA, patients with a large infarct were more often in the mRS 2-6 group (28.4\%) than in the mRS $0-1$ group $(8.1 \%$, $\mathrm{p}=0.003)$. IV rt-PA was administered to 8 of 43 patients $(18.6 \%)$ with hyper-acute ischemic stroke in the mRS $0-1$ group and 27 of 40 patients $(67.5 \%)$ in the mRS $2-6$ group $(\mathrm{p}<0.001)$.

Factors associated with mRS $0-1$ were analyzed using multivariate logistic regression analysis. NIHSS score was considered a confounding factor and excluded from analysis. The only factor independently associated with mRS $0-1$ was KPSS $<3$ (odds ratio, 3.0; 95\% confidence interval, $1.2-7.3, \mathrm{p}=0.015$; fig. 2).

\section{Discussion}

The present results confirmed good validity for the initial KPSS score in predicting clinical outcome 3 months after symptom onset, which was assessed using the mRS to quantify functional activities and global handicap of daily living. These findings are supportive of the 


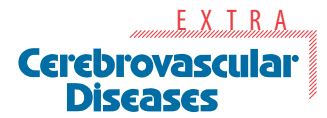

\begin{tabular}{l|l}
\hline Cerebrovasc Dis Extra 2011;1:28-35 \\
\hline $\begin{array}{l}\text { DOI: 10.1159/000327154 } \\
\text { Published online: April 14, } 2011\end{array}$ & $\begin{array}{l}\text { @ 2011 S. Karger AG, Basel } \\
\text { www.karger.com/cee }\end{array}$ \\
\hline
\end{tabular}

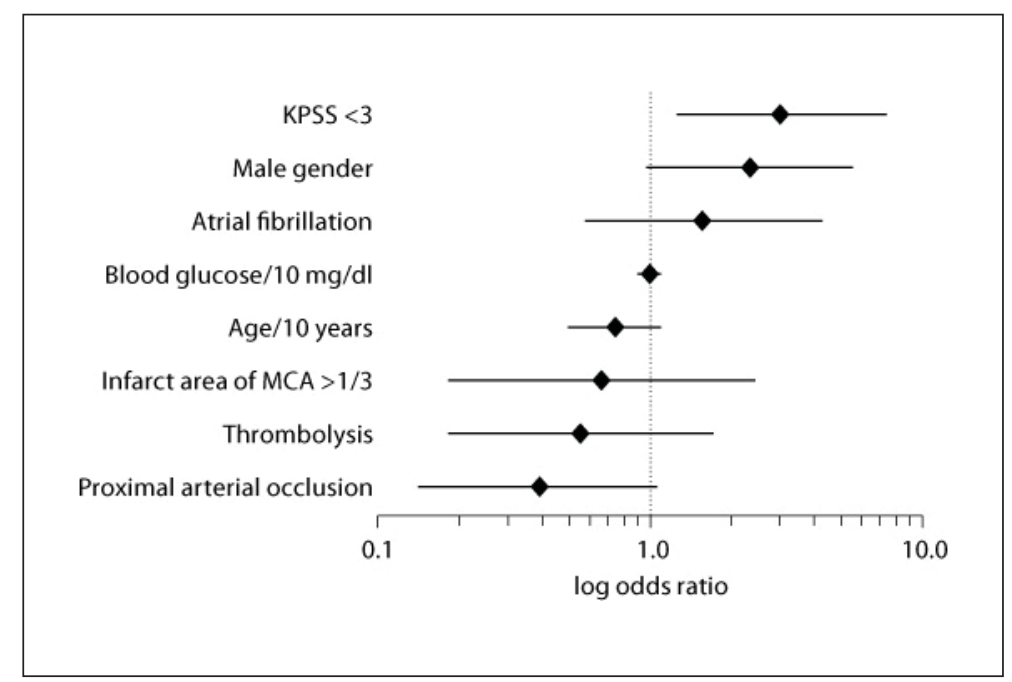

Fig. 2. A forest plot diagram showing factors for mRS $0-1$ at 3 months after symptom onset in a multivariate logistic regression model.

previous finding that the LAMS correlates with functional outcome at 3 months as well as the full NIHSS [7]. The reason for this relationship may be as follows. First, clinical prognosis of stroke patients was strongly associated with initial stroke severity, which is generally assessed using the NIHSS [17]. Indeed, because an excellent correlation was evident between KPSS and NIHSS scores in our series, KPSS score $<3$ should be considered as a factor independently associated with a favorable outcome 3 months after onset.

Our assessment of stroke severity was performed in a straightforward manner by paramedics in the prehospital field. Actually, the KPSS, LAMS, sNIHSS, and 3-ISS are simple scoring systems [7-9], but results from previous investigations might have been influenced by the setting (field in the present study vs. emergency room in the other three studies) or examiner (paramedics in this study vs. physicians in other studies). We thus consider that this examination using the KPSS in a realistic situation should be sufficiently robust to confirm the potential for PSSSs.

What is the essential information for assessing possible stroke patients as having mild or severe symptoms in prehospital scenarios? Two applications should be outlined in this regard. First, when neurological symptoms deteriorate during transportation, an urgent and precise diagnosis in a stroke center is required to assess and rescue vulnerable brain tissue. Patients with a PSSS possibly indicating large artery occlusion may sometimes be best transferred directly to a comprehensive stroke center where endovascular recanalization interventions may be available [9]. The KPSS will thus allow adequate decision-making and transport in prehospital settings. Furthermore, pretreatment evaluation of stroke severity using the KPSS may predict long-term outcomes of stroke patients and has great potential as an important initial value for prehospital clinical research. Although we have no supporting data for this issue from previous studies, one proposal is that a certified assessment of pretreatment stroke severity will be a future requirement for prehospital stroke clinical treatment trials investigating the development of adequate transportation systems.

An important limitation of this study was the analysis of only a single dichotomized outcome using mRS. Then, substantial interrater variability of mRS measurement may be present because we are unable to conduct a structured assessment tool in order to reduce 
mRS variability. To confirm the utility of the KPSS in a Japanese prehospital setting, prospective, multicenter trials should be extensively conducted.

In conclusion, the KPSS shows promise as an excellent tool enabling paramedics to quantify stroke severity in the prehospital setting. Prehospital assessment of stroke severity using the KPSS may help to predict a favorable outcome in acute cerebral ischemia.

\section{Acknowledgment}

This study was supported by a Kawasaki Medical School Project grant (k0104274) from the Kawasaki Medical School, Japan. The authors thank the Kurashiki Fire Bureau for supporting this study.

\section{Disclosure Statement}

The authors have no competing interest to report.

\section{References}

1 Tissue plasminogen activator for acute ischemic stroke: The National Institute of Neurological Disorders and Stroke rt-PA Stroke Study Group. N Engl J Med 1995;333:1581-1587.

-2 Schroeder EB, Rosamond WD, Morris DL, Evenson KR, Hinn AR: Determinants of use of emergency medical services in a population with stroke symptoms: the Second Delay in Accessing Stroke Healthcare (DASH II) Study. Stroke 2000;31:2591-2596.

-3 Iguchi Y, Wada K, Shibazaki K, Inoue T, Ueno Y, Yamashita S, Kimura K: First impression at stroke onset plays an important role in early hospital arrival. Intern Med 2006;45:447-451.

-4 Kothari RU, Pancioli A, Liu T, Brott T, Broderick J: Cincinnati Prehospital Stroke Scale: reproducibility and validity. Ann Emerg Med 1999;33:373-378.

-5 Kidwell CS, Starkman S, Eckstein M, Weems K, Saver JL: Identifying stroke in the field. Prospective validation of the Los Angeles Prehospital Stroke Screen (LAPSS). Stroke 2000;31:71-76.

6 Kimura K, Inoue T, Iguchi Y, Shibazaki K: Kurashiki Prehospital Stroke Scale. Cerebrovasc Dis 2008; 25:189-191.

-7 Llanes JN, Kidwell CS, Starkman S, Leary MC, Eckstein M, Saver JL: The Los Angeles Motor Scale (LAMS): a new measure to characterize stroke severity in the field. Prehosp Emerg Care 2004;8:4650 .

8 Tirschwell DL, Longstreth WT Jr, Becker KJ, Gammans RE Sr, Sabounjian LA, Hamilton S, Morgenstern LB: Shortening the NIH Stroke Scale for use in the prehospital setting. Stroke 2002;33:28012806.

-9 Singer OC, Dvorak F, du Mesnil de Rochemont R, Lanfermann H, Sitzer M, Neumann-Haefelin T: A simple 3-item stroke scale: comparison with the National Institutes of Health Stroke Scale and prediction of middle cerebral artery occlusion. Stroke 2005;36:773-776.

-10 Iguchi Y, Kimura K, Watanabe M, Shibazaki K, Aoki J: Utility of the Kurashiki Prehospital Stroke Scale for hyperacute stroke. Cerebrovasc Dis 2010;31:51-56.

-11 Nazliel B, Starkman S, Liebeskind DS, Ovbiagele B, Kim D, Sanossian N, Ali L, Buck B, Villablanca P, Vinuela F, Duckwiler G, Jahan R, Saver JL: A brief prehospital stroke severity scale identifies ischemic stroke patients harboring persisting large arterial occlusions. Stroke 2008;39:2264-2267.

12 Aruga T (ed): PSLS Guide Book. Tokyo, Herusu Shyuppan, 2007, pp 19-22.

-13 Yamaguchi T, Mori E, Minematsu K, Nakagawara J, Hashi K, Saito I, Shinohara Y, Japanese Alteplase Clinical Trial (J-ACT) Group: Alteplase at $0.6 \mathrm{mg} / \mathrm{kg}$ for acute ischemic stroke within 3 hours of onset: Japan Alteplase Clinical Trial (J-ACT). Stroke 2006;37:1810-1815. 
Iguchi et al.: KPSS and Outcome

-14 Nighoghossian N, Hermier M, Adeleine P, Derex L, Dugor JF, Philippeau F, Ylmaz H, Honnorat J, Dardel P, Berthezene Y, Froment JC, Trouillas P: Baseline magnetic resonance imaging parameters and stroke outcome in patients treated by intravenous tissue plasminogen activator. Stroke 2003;34: 458-463.

-15 Appelros P, Nydevik I, Viitanen M: Poor outcome after first-ever stroke: predictors for death, dependency, and recurrent stroke within the first year. Stroke 2003;34:122-126.

- 16 Saposnik G, Di Legge S, Webster F, Hachinski V: Predictors of major neurologic improvement after thrombolysis in acute stroke. Neurology 2005;65:1169-1174.

- 17 Frankel MR, Morgenstern LB, Kwiatkowski T, Lu M, Tilley BC, Broderick JP, Libman R, Levine SR, Brott T: Predicting prognosis after stroke: a placebo group analysis from the National Institute of Neurological Disorders and Stroke rt-PA Stroke Trial. Neurology 2000;55:952-959. 